\title{
Effect of Organic Fertilization and AMF Inoculation on Yield and Floral Quality Parameters of Common Marigold
}

\author{
Panayiota PAPASTYLIANOU ${ }^{1 *}$, George STAVROPOULOS ${ }^{1}$, Iordanis SAMANIDIS ${ }^{2}$ Dimitrios BILALIS $^{1}$ \\ ${ }^{1}$ Agricultural University of Athens, Department of Crop Science, Iera Odos 75, 11855, Athens, Greece \\ ${ }^{2}$ Korres Natural Products SA \\ *)Corresponding author, e-mail: ppapastyl@aua.gr
}

BulletinUASVM Horticulture 74(1) / 2017

Print ISSN 1843-5254, Electronic ISSN 1843-5394

DOI:10.15835/buasvmcn-hort:12640

\begin{abstract}
In Greece common marigold is one of the major medicinal plants widely used in cosmetics, perfumes and the pharmaceutical industry. A field experiment was conducted at Komotini, Greece, to compare the effect of organic and conventional fertilization combined with arbuscular mycorrhizal fungi inoculation on yield and floral qualitative characteristics of the common marigold during the 2015 growing season. The experiment was set up as a split plot design with three replicates, three main plots (fertilization treatments, inorganic, organic and untreated) and two sub-plots (addition/non-addition of commercial mycorrhiza of the genus Glomus spp.). Floral fresh and dry weight as well as total phenolic and flavonoid content of the dried flowers were recorded. Data analysis confirmed no significant correlation between fresh/dry floral yield, total phenolic and flavonoid content of the dried flowers and type of fertilization. The results also demonstrate a tendency of increase of the fresh or dry weight of the flowers when the commercial mycorrhiza is applied but it is not statistically significant.
\end{abstract}

Keywords: arbuscular mycorrhiza, calendula officinalis, fertilization, flavonoids, polyphenols

\section{Introduction}

The common marigold (Calendula officinalis L.) is a commercially cultivated annual aromatic and pharmaceutical plant because of the wide range of its potential pharmaceutical and cosmetic usages, since extensive investigation has confirmed the abundance of various active phytochemical compounds responsible for the properties of the plant. The species have been reported to contain a variety of phytochemicals, including carbohydrates, lipids, phenolic compounds, steroids, terpenoids, tocopherols, carotenoids and quinones with potential health benefits (Muley et al., 2009). On the other hand only a limited scientific knowledge has been gathered related to the optimum cultural practices, the efficacy of organic cultivation and the correlation of production methods with the quality characteristics of this species. The aim of this study was to evaluate the effects of organic and conventional fertilization combined with arbuscular mycorrhizal fungi inoculation on yield and floral qualitative characteristics of the common marigold during the 2015 growing season.

\section{Materials and methods}

The research was carried out at a private organic farm, certified as "organic" since 1995, located in Komotini (northern Greece: latitude $41^{\circ} 02^{\prime} \mathrm{N}$, longitude $25^{\circ} 12^{\prime} \mathrm{E}$, altitude $16 \mathrm{~m}$ above sea level) from April to October 2015. The soil was sandy clay ( $40.5 \%$ clay, $9.0 \%$ silt and $50.5 \%$ sand) with pH 7.43, $\mathrm{NO}_{3}-\mathrm{N} 12.0 \mathrm{mg} \mathrm{kg}^{-1}$ soil, available $\mathrm{P}$ $18.0 \mathrm{mg} \mathrm{kg}^{-1}$ soil, available $\mathrm{K} 460 \mathrm{mg} \mathrm{kg}^{-1}$ soil and $1.61 \%$ organic matter. The experiment was set up as a split plot design with three replicates, three main plots (fertilization treatments, inorganic, organic and untreated) and two sub-plots 

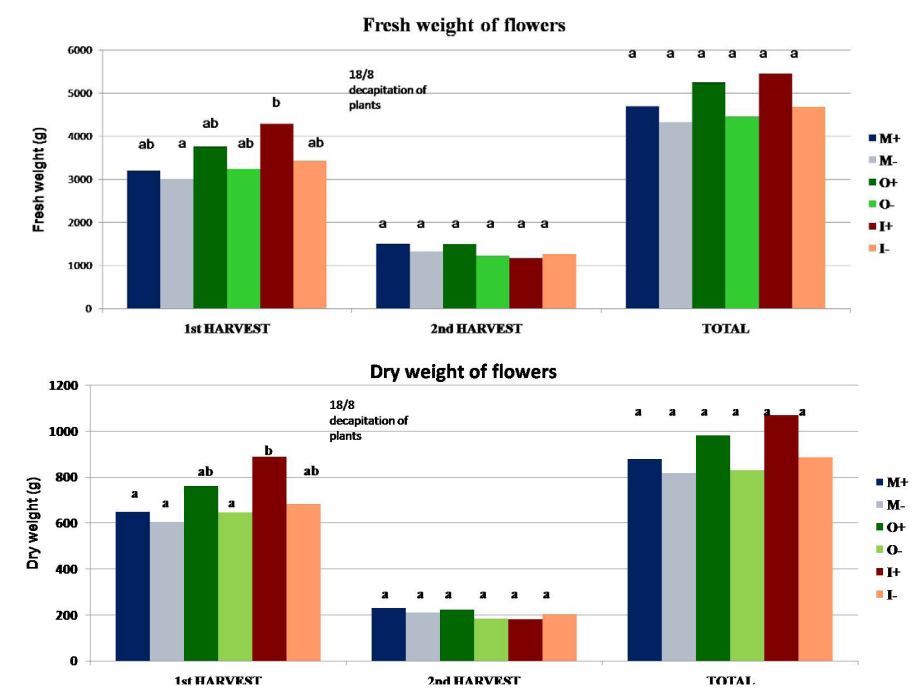

Fig. 1. and 2. Response of fresh and dry weight of flowers to fertilization and mycorrhiza treatments. Different low case letters indicate significant differentes $(\mathrm{p}<0.05)$.

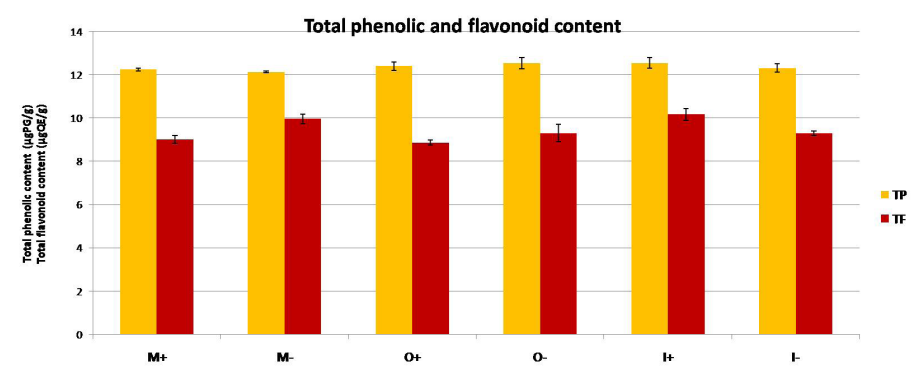

Fig. 3. Total phenolic (TP) and flavonoid (TF) content of marigold flowers.

(addition/non-addition of commercial mycorrhiza of the genus Glomus spp.). Seeds of a local cultivar from a native wild population were placed in a plastic tray containing a mixture of sand:organic substrate:soil in the proportion of 1:1:1. When the seedlings reached $15 \mathrm{~cm}$, they were replanted in the experimental field on 10 May. The experimental plots were $7 \mathrm{~m} \times 2 \mathrm{~m}$ and consisted of 2 rows $1 \mathrm{~m}$ apart. For both organic and inorganic fertilization the fertilizers applied provided the soil with 10 units of $\mathrm{N} / \mathrm{P} / \mathrm{K}$. Marigold inflorescences were harvested in the full bloom phase, every 7 days from $25 / 6$ to $22 / 10$. Floral fresh and dry weight as well as total phenolic and flavonoid content of the dried flowers were recorded. The total phenolic content of the extract was determined by the FolinCiocalteu method (Blainski et al., 2013) and the aluminum chloride colorimetric method was used for the determination of the total flavonoid content of the sample (Chang et al., 2002). The data were subjected to statistical analysis according to the split-plot design (STATGRAPHICS Plus 5.1 logistic package). Differences between treatment means were compared at $\mathrm{P}=5 \%$ with ANOVA in order to find the statistically significant differences.

\section{Results and discussion}

The results indicate no statistically significant differences in the fresh/dry floral yield, total phenolic and flavonoid content of the dried flowers under the different treatments of fertilization (Fig. 1,2 and 3). The results also demonstrate a tendency of increase of the fresh or dry weight of the flowers when commercial mycorrhiza is applied but it is not statistically significant (Fig. 1 and 2). Our results are in accordance with previous studies (Hosseinzadah et al., 2011, Fernandes et al., 2013). In addition, the results obtained by Borella et al. (2011) indicated that calendula exhibits hardiness and adapts well to the soils that are relatively poor in organic material, but when organic fertilization was applied, it could prolongate the vegetative 
development of this species. However, the organic fertilizer releases nutrients to the plants slower and in a higher constant manner, bringing the benefits of chemical and physical properties of the soil. The organic fertilization improves the soil structure, aeration, drainage and water retention. Thus, total flavonoid content in C. officinalis might depend on the cultivar and the environmental conditions during the cultivation period.

\section{Conclusion}

Based on the results obtained in the first experimental year, we demonstrated that organic fertilization of the $C$. officinalis is not inferior to inorganic fertilization of the specific species for the characteristics of fresh/dry flower mass, total flavonoid and polyphenolic content. This statement needs to be confirmed by future research regarding the effect of fertilization management on yield and floral quality parameters of common marigold.

\section{REFERENCES}

1. Blainski A, Lopes GC, Mello JCP (2013). Application and Analysis of the Folin Ciocalteu Method for the Determination of the Total Phenolic Content from Limonium Brasiliense L. Molecules18:6852-6865.

2. Borella JC, Ribeiro NS, Freato AMR, Mazzo KF, Barbosa DM (2011). Influencia da adubacao e da cobertura morta na produtividade e no teor de flavonoides de Calendula officinalis L. (Asteraceae). Rev Bras Plantas Med 13:235239.

3. Chang CC, Yang MH, Wen HM, Chern JC (2002). Estimation of total flavonoid content in propolis by two complementary colorimetric methods. J Food Drug Anal 10:178-182.

4. Fernandes EFA, Meloni F, Borella JC, Lopesa, NP (2013). Effect of fertilisation and harvest period on polar metabolites of Calendula officinalis. Rev Bras Farmacogn 23:731-735.

5. Hosseinzadah F, Aryan Satei A, Mahmoud Reza Ramezanpour MR (2011). Effects of Mycorhiza and Plant Growth Promoting Rhizobacteria on Growth, Nutrients Uptake and Physiological Characteristics in Calendula officinalis L. Middle-East J Sci Res 8:947-953.

6. Muley BP, Khadabadi SS, Banarase NB (2009). Phytochemical Constituents and Pharmacological Activities of Calendula officinalis Linn (Asteraceae): A Review. Trop J Pharm Res 8:455-465. 